\title{
Terahertz Wave Modulation in PCBM-Deposited Metallic Slit Arrays
}

\author{
Joong Wook Lee* \\ Department of Physics and Optoelectronics Convergence Research Center, Chonnam National University, Gwangju 61186, Korea
}

Received December 20, 2018; accepted December 28, 2018

\begin{abstract}
The properties of terahertz $(\mathrm{THz})$ wave modulation were investigated in organic-deposited metallic slit arrays fabricated on a Si substrate. The organic thin layer (fullerene derivative [6,6]-phenyl-C61-butyric acid methyl ester, also known as PCBM) acts as an active layer controlled by the incident light; thus, modulating the transmission of the incident $\mathrm{THz}$ waves. This is due to the photoexcited electron transfer from the silicon substrate to the PCBM layer, which results in the organic layer becoming metallic. The metallic slit arrays show plasmonic properties, such as wavelengthdependent transmission, which depends on the structural arrangements of the slits. When the polarization of the incident $\mathrm{THz}$ waves is parallel with the direction of the metallic slits, a cut-off frequency clearly appears, while for perpendicular polarization, the transmission is considerably enhanced by the plasmonic property. The hybrid structures of organic-metallic slits deposited on a silicon substrate enable the active control of the spoof surface plasmonmediated transmission of $\mathrm{THz}$ waves. This finding can help realize multi-functional $\mathrm{THz}$ devices and design spectrally controllable $\mathrm{THz}$ wave modulators for $\mathrm{THz}$ sensing or imaging systems.
\end{abstract}

Keywords: Terahertz wave, Organic, Modulation

\section{Introduction}

The development of terahertz (THz) devices has attracted significant research interest owing to their broad application in various fields, including $\mathrm{THz}$ spectroscopy, imaging, and communications [1-3]. In particular, active $\mathrm{THz}$ modulators have been extensively investigated for their use as active optical filters, active beam splitters, active mirrors, switches, and transistors. Various technologies have been applied to achieve active modulation of $\mathrm{THz}$ wave transmission. Different materials and structures, such as metamaterials, vanadium dioxide $\left(\mathrm{VO}_{2}\right)$, graphene, and semiconductor nanostructures, have been investigated by several research groups [4-9].

Recently, organic-based hybrid structures were suggested for the active control of the transmission of $\mathrm{THz}$ waves [10]. These structures have shown excellent properties and remarkable performance in terms of modulation efficiency, dynamic range, and functionality. Since our group reported organic-based active $\mathrm{THz}$ modulators with a modulation efficiency of $55 \%$ in 2011 for the first time, the modulation efficiency had been steadily improved. In 2017, Yoo et al. demonstrated the optimal conditions for higher modulation efficiency in trilayer hybrid structures based on fullerene derivative [6,6]-phenyl-C61-butyric

*Corresponding author

E-mail: leejujc@chonnam.ac.kr acid methyl ester (PCBM) [11]. In the present study, the modulation efficiency reached nearly $100 \%$, achieved by optimizing the conditions of the annealing temperature, the intensity and wavelength of the excitation laser, the thickness of the organic layers, and the arrangement of the organic thin films. Several other research groups have reported that the active modulation of $\mathrm{THz}$ waves can be achieved by using different organic materials, polymers, or even perovskites [12-16]. Although these studies provide useful methods for improving the modulation efficiency, spectral broadness, and structural simplicity for convenient fabrication, specific functionalities, such as a selective active modulation, need to be further investigated for various applications of active $\mathrm{THz}$ modulators.

In this paper, we present organic-deposited metallic slit arrays fabricated on a $\mathrm{Si}$ substrate used for active $\mathrm{THz}$ modulators. The organic thin layer, which acts as an electron acceptor, is made of PCBM. The photoexcited electrons on the Si substrate are injected into the PCBM thin layer due to the highest occupied molecular orbitallowest unoccupied molecular orbital (HOMO-LUMO) energy level relationship between the Si and the PCBM. The transmission rate of $\mathrm{THz}$ waves is decreased by the contribution of the long-lived carriers due to the separation of electrons and holes. This indicates that the PCBM thin layer becomes more metallic under photoexcitation. A plasmonic structure, consisting of periodic arrays of slits sandwiched between the Si substrate and PCBM thin layer, 
is applied to systematically realize wavelength- and polarization-dependent modulation of $\mathrm{THz}$ waves. The organic-metal-Si hybrid structures can be useful for designing actively controllable multi-functional $\mathrm{THz}$ devices and THz systems, such as spectroscopy, sensing, and imaging, requiring a complex composition of several different devices with various functions.

\section{Experimental details}

The plasmonic structure, consisting of periodic arrays of metallic slits, was fabricated on a Si substrate with the thickness of $500 \mu \mathrm{m}$ and high resistivity of up to $1.0 \times 10^{5}$ $\Omega \cdot \mathrm{cm}$, by conventional photolithography techniques. Figure 1(a) shows a microscopic image of the sample and an enlarged image with the details of the period and the width of the metallic slits. The PCBM molecules with purity higher than $99.5 \%$, purchased from Sigma-Aldrich (product number: 684449), were deposited on the plasmonic-Si hybrid structure, using a spin coating method. Figure 1(b) shows the molecular structure of the PCBM molecule. The thickness of the PCBM thin layer was $\sim 150 \mu \mathrm{m}$, achieved by varying the rotation speed of the spin coater and the organic concentration. Figure 1(c) shows a schematic diagram of the $\mathrm{THz}$ wave modulation experiment. The $\mathrm{THz}$ waves are incident on the side of the PCBM thin layer, and the active sample area where the $\mathrm{THz}$ waves and optical beams overlap with each other is fixed by a pinhole with the diameter of $3 \mathrm{~mm}$. The dynamics of the photoexcited carriers at the interface between the PCBM and Si depends on the HOMO-LUMO energy level diagram shown in Fig. 1(d).

The modulated $\mathrm{THz}$ transmission was measured using a
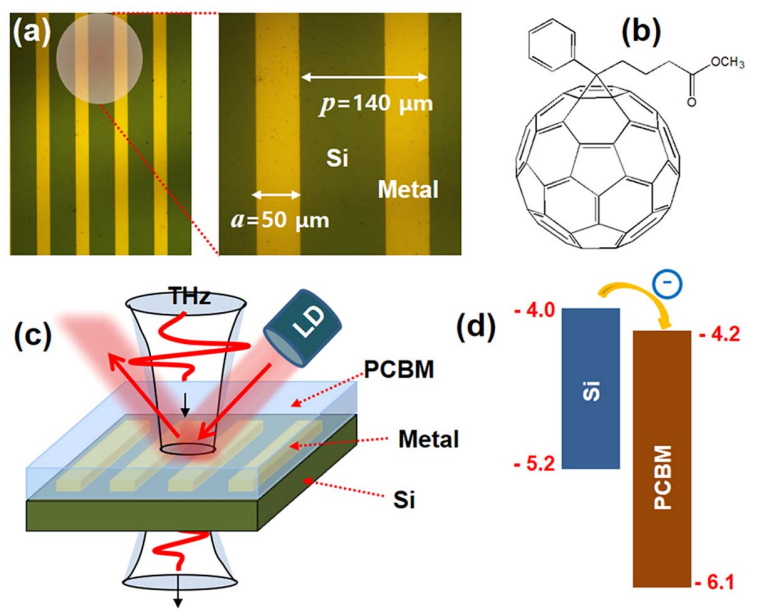

Figure 1. (a) Microscopic images of the metallic slit arrays deposited on the Si substrate. The period and the width of slits are 140 and $50 \mu \mathrm{m}$, respectively. (b) Structure of a PCBM molecule. (c) Schematic diagram of the THz wave modulation experiment. The optical beam for photoexcitation is incident on the PCBM/metallic slit arrays/Si hybrid structure. (d) HOMO-LUMO energy level diagram of the PCBM/Si bilayer structure.
$\mathrm{THz}$ time-domain spectroscopy system. The $\mathrm{THz}$ waves were generated by a (100) p-type InAs wafer and the transmissions were measured by a photoconductive antenna method [17]. Using a cw laser diode with the wavelength of $785 \mathrm{~nm}$ and laser power up to $220 \mathrm{~mW}$, the electrons and holes were generated on the active sample area with a diameter of $\sim 3 \mathrm{~mm}$, formed by focusing the optical beam. The transmission time-domain signals measured with and without photoexcitation were compared and the spectral amplitudes were obtained by Fouriertransform method.

\section{Results and discussion}

Figure 2 shows the transmission spectra of $0^{\circ}$ transverse magnetic (TM) and $90^{\circ}$ transverse electric (TE) polarized $\mathrm{THz}$ waves, measured on the plasmonic-Si hybrid structure (without the PCBM thin layer), with and without photoexcitation, respectively. In the geometric regime, the wave-guided modes originated from the boundary conditions due to the width of metallic slits can be observed for the TE polarization. In this case, the effect of surface plasmons does not appear, while for the TM polarization, the transmission spectra measured at $0^{\circ}$ polarization indicate the enhancement of the transmitted $\mathrm{THz}$ waves in the subwavelength limit [18]. For the $\mathrm{Si}$ substrate, the first waveguide mode appearing at $90^{\circ}$ polarization is determined by the equation $\lambda_{r}=2 n(p-a)$, where $n$ is the refractive index of $\mathrm{Si}, a$ is the width of metallic slits, and $p$ is the period of metallic slit arrays. Therefore, the cut-off frequency, specifically the first waveguide mode, appears near $0.6 \mathrm{THz}$, as shown by the red curve in Fig 2. On the contrary, for the TM polarization, the transmitted $\mathrm{THz}$ waves are enhanced due to the plasmonic effect of surface charges as well black curve, Fig. 2. Under the condition of photoexcitation, the transmissions of $\mathrm{THz}$ waves are reduced with consistent

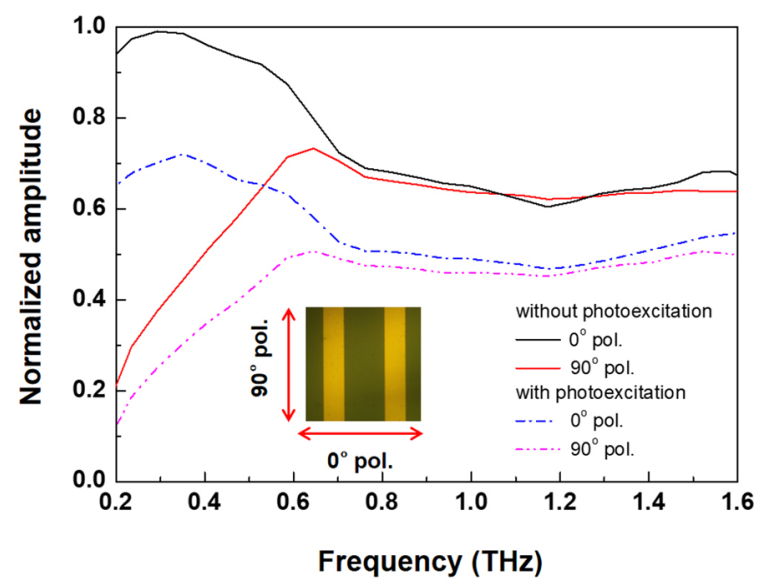

Figure 2. Measured amplitude transmission spectra of the plasmonic-Si hybrid structure for $0^{\circ}$ (TM) and $90^{\circ}$ (TE) polarizations, with or without photoexcitation for each polarization. 
differences throughout all frequency ranges. This is solely due to the effect of the photoexcited carriers on the $\mathrm{Si}$ substrate.

The dependence of laser power of the optical beam for photoexcitation on the modulation efficiency was investigated. Figure 3 shows the measured transmission amplitude spectra of the PCBM-plasmonic slits-Si trilayer hybrid structure, for $0^{\circ}$ [Fig. 3(a)] and $90^{\circ}$ [Fig. 3(b)] polarizations. As the laser power increases, the transmission rapidly decreases; in other words, the modulation efficiency dramatically increases, compared with those of the plasmonic slits-Si bilayer structure. The experimental results reveal that the remarkable change in the transmission of $\mathrm{THz}$ waves strongly depends on the presence of the PCBM layer. This implies that the increase in the modulation efficiency is associated with the carrier diffusion and injection at the interface between the PCBM thin film and the Si substrate.

When the optical beam with a center wavelength of $785 \mathrm{~nm}$ is incident on the $\mathrm{Si}$ substrate, photoexcited electrons and holes are generated. Based on the HOMOLUMO energy level shown in Fig. 1(d), the photoexcited electrons are injected from the Si substrate into the PCBM thin layer. The PCBM thin layer becomes more metallic
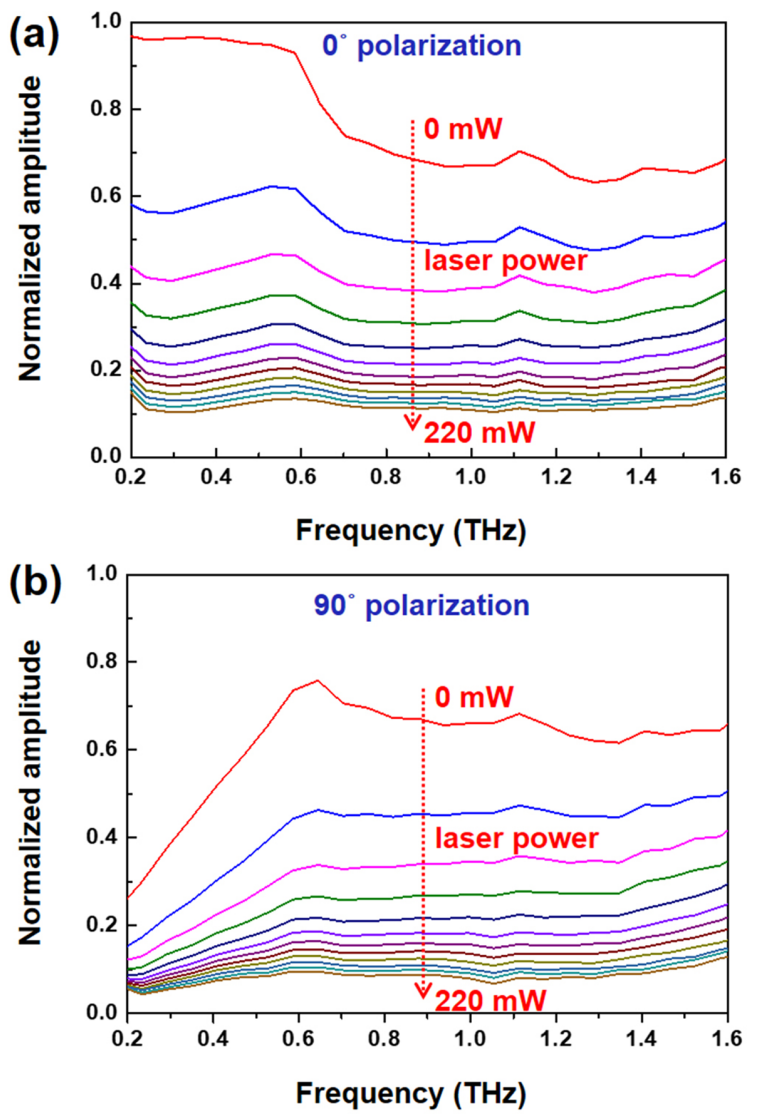

Figure 3. Measured amplitude transmission spectra of the PCBM-plasmonic-Si hybrid structure by varying the laser power of the optical beams, for (a) $0^{\circ}$ (TM) and (b) $90^{\circ}$ (TE) polarizations. due to the long-lived carriers due to the separation of the free electrons and holes; consequently the transmission of the incident $\mathrm{THz}$ waves is greatly reduced.

To quantitatively analyze the modulation efficiency, the spectral intensity modulation efficiency is defined as [12]

$$
M=\frac{\int\left|E_{u n}(\omega)\right|^{2} d \omega-\int\left|E_{e x}(\omega)\right|^{2} d \omega}{\int\left|E_{u n}(\omega)\right|^{2} d \omega},
$$

where $E_{u n}$ and $E_{e x}$ represent the electric field amplitude spectra measured with and without photoexcitation, respectively [20]. To analyze the characteristics of the frequency-dependent modulation of $\mathrm{THz}$ waves, the value of the peak modulation efficiency obtained at a specific frequency is given by

$$
M_{p}=\frac{\left|E_{u n}\left(\omega_{0}\right)\right|^{2}-\left|E_{e x}\left(\omega_{0}\right)\right|^{2}}{\left|E_{u n}\left(\omega_{0}\right)\right|^{2}} .
$$

Figure 4(a) shows the spectral intensity modulation efficiency, $M$, of the THz wave transmission, plotted as a function of the laser power of the optical beam for photoexcitation. The black and red curves indicate the $M$ values for $0^{\circ}$ (TM) and $90^{\circ}$ (TE) polarizations, respectively. For both cases, the modulation efficiencies
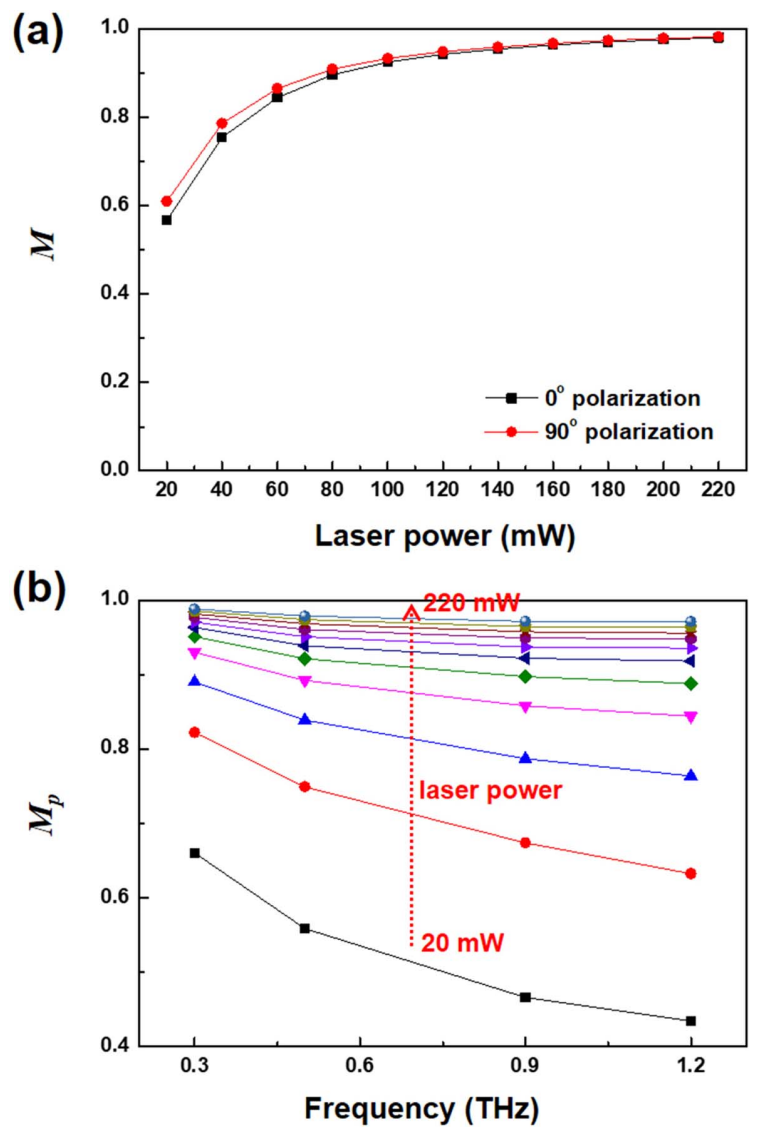

Figure 4. (a) Modulation efficiency of THz wave transmission for the PCBM-plasmonic slits-Si trilayer hybrid structure plotted as a function of the laser power of the optical beams for photoexcitation, for $0^{\circ}$ (black squares) and $90^{\circ}$ (red circles) polarizations. (b) Peak modulation efficiencies at different laser power values as a function of frequency. 
increase with the increase in the laser power of the optical beam. The modulation efficiency reaches nearly $60 \%$ at only $20 \mathrm{~mW}\left(0.28 \mathrm{~W} / \mathrm{cm}^{2}\right)$ and approximately $90 \%$ at only $100 \mathrm{~mW}\left(1.4 \mathrm{~W} / \mathrm{cm}^{2}\right)$. This implies that regardless of the rather complex structure of the PCBM-plasmonic slits-Si hybrid, compared to that of organic-Si bilayers, the modulation efficiency is highly sensitive to the density of photoexcited carriers accumulated in the PCBM thin layer.

To quantitatively analyze the frequency-dependent properties of the modulation efficiency, we calculated the $M_{p}$ values at frequencies of $0.3,0.6,0.9$ and $1.2 \mathrm{THz}$, as shown in Fig. 4(b). As expected, the modulation efficiencies increase with the increase in the laser power of the optical beam over all frequencies. However, the rate of increase of the modulation efficiency is different at different frequencies, but still depending on the laser power. At a fixed laser power of $20 \mathrm{~mW}$, the modulation efficiency at longer wavelengths (lower frequencies) is higher than those at shorter wavelengths. Apparently, the behavior of the PCBM thin layer becomes similar to that of metal more easily at longer wavelengths when the carrier concentration is relatively low. This is similar to that of the plasma frequency, determining the spectral boundary exhibiting metallic properties, which shifts to higher frequencies with the increase of carrier concentration. Understanding the wavelength-dependent modulation efficiency can be useful for the design of active $\mathrm{THz}$ modulators with frequency-selective function.

\section{Conclusions}

In this study, optically controllable $\mathrm{THz}$ wave modulators based on a PCBM-plasmonic metamaterial-Si trilayer hybrid structure were investigated. The decrease in the transmission of $\mathrm{THz}$ waves was due to the PCBM thin film, which acts as an active layer, and the characteristics of the wavelength-dependent modulation was explained by the contribution of the plasmonic metamaterial structure based on the periodic arrays of metallic slits. The cut-off frequency was clearly observed at $0^{\circ}$ (TE) polarization, while the enhancement of the transmission of $\mathrm{THz}$ waves occurred at $90^{\circ}(\mathrm{TM})$ polarization. In all cases, the modulation of the transmission of the $\mathrm{THz}$ waves was realized throughout the $\mathrm{THz}$ frequency range, despite that the modulation efficiency slightly depends on the wavelength of the incident $\mathrm{THz}$ waves. The understanding of these phenomena can provide practical benefits in the design of spectrally controllable $\mathrm{THz}$ modulators for advanced $\mathrm{THz}$ sensing or imaging systems and in realizing multi-functional $\mathrm{THz}$ devices.

\section{Acknowledgements}

This research was supported by the Basic Science Research Program through the National Research Foundation of Korea (NRF), funded by the Ministry of Education, Science and Technology and the Korea government (MSIP) (NRF-2016R1D1A1B03935241). This study was financially supported by the Chonnam National University (Grant number: 2017-2754).

\section{References}

[1] D. M. Mittleman, M. Gupta, R. Neelamani, R. G. Baraniuk, J. V. Rudd, and M. Koch, Appl. Phys. B 68, 1085 (1999).

[2] P. H. Siegel, IEEE Trans. Microwave Theory Tech. 50, 910 (2002).

[3] B. Ferguson and X. C. Zhang, Nat. Mater. 1, 26-33 (2002).

[4] H. T. Chen, W. J. Padilla, J. M. O. Zide, A. C. Gossard, A. J. Taylor, and R. D. Averitt, Nature 444, 597 (2006).

[5] S. H. Lee, M. Choi, T. T. Kim, S. Lee, M. Liu, X. Yin, H. K. Choi, S. S. Lee, C. G. Choi, S. Y. Choi, X. Zhang, and B. Min, Nat. Mater. 11, 936 (2012).

[6] D. J. Hilton, R. P. Prasankumar, S. Fourmaux, A. Cavalleri, D. Brassard, M. A. El Khakani, J. C. Kieffer, A. J. Taylor, and R. D. Averitt, Phys. Rev. Lett. 99, 226401 (2007).

[7] M. Seo, J. Kyoung, H. Park, S. Koo, H. Kim, H. Bernien, B. J. Kim, J. H. Choe, Y. H. Ahn, H. T. Kim, N. Park, Q. H. Park, K. Ahn, and D. S. Kim, Nano Lett. 10, 2064 (2010).

[8] B. Sensale-Rodriguez, R. Yan, M. M. Kelly, T. Fang, K. Tahy, W. S. Hwang, D. Jena, L. Liu, and H. G. Xing, Nat. Commun. 3, 780 (2012).

[9] X. Wu, X. Pan, B. Quan, and L. Wang, Appl. Phys. Lett. 103, 121112 (2013).

[10] H. K. Yoo, C. Kang, Y. W. Yoon, H. J. Lee, J. W. Lee, K. Lee, and C. S. Kee, Appl. Phys. Lett. 99, 061108 (2011).

[11] H. K. Yoo, H. Lee, K. Lee, C. Kang, C. S. Kee, I. W. Hwang, and J. W. Lee, AIP Adv. 7, 105008 (2017).

[12] H. K. Yoo, Y. W. Yoon, K. Lee, C. Kang, C. S. Kee, I. W. Hwang, and J. W. Lee, Appl. Phys. Lett. 105, 011115 (2014).

[13] T. He, B. Zhang, J. Shen, M. Zang, T. Chen, Y. Hu, and Y. Hou, Appl. Phys. Lett. 106, 053303 (2015).

[14] L. Zhong, B. Zhang, T. He, L. Lv, Y. Hou, and J. Shen, Appl. Phys. Lett. 108, 103301 (2016).

[15] K. S. Lee, R. Kang, B. Son, D. Y. Kim, N. E. Yu, and D. K. Ko, Sci. Rep. 6, 37912 (2016).

[16] T. Matsui, H. Mori, Y. Inose, S. Kuromiya, K. Takano, M. Nakajima, and M. Hangyo, Jpn. J. Appl. Phys. 55, 03DC12 (2016).

[17] J. W. Lee, J. K. Yang, I. B. Sohn, H. K. Choi, C. Kang, and C. S. Kee, Opt. Eng. 51, 119002 (2012).

[18] J. W. Lee, T. H. Park, P. Nordlander, and D. M. Mittleman, Opt. Exp. 17, 12660 (2009). 